\title{
Neutrosophic Exponential Distribution: Modeling and Applications for Complex Data Analysis
}

\author{
Wen-Qi Duan, ${ }^{1,2}$ Zahid Khan $\mathbb{D}^{\circ},{ }^{3}$ Muhammad Gulistan $\mathbb{D}^{3},{ }^{3}$ and Adnan Khurshid ${ }^{1}$ \\ ${ }^{1}$ School of Business, Taizhou University, Taizhou 318000, China \\ ${ }^{2}$ College of Economics and Management, Zhejiang Normal University, Jinhua 321004, China \\ ${ }^{3}$ Department of Mathematics and Statistics, Hazara University, Mansehra, Pakistan \\ Correspondence should be addressed to Zahid Khan; zahidkhan@hu.edu.pk
}

Received 10 August 2021; Accepted 13 September 2021; Published 28 September 2021

Academic Editor: Zeljko Stevic

Copyright ( 2021 Wen-QI Duan et al. This is an open access article distributed under the Creative Commons Attribution License, which permits unrestricted use, distribution, and reproduction in any medium, provided the original work is properly cited.

\begin{abstract}
The exponential distribution has always been prominent in various disciplines because of its wide range of applications. In this work, a generalization of the classical exponential distribution under a neutrosophic environment is scarcely presented. The mathematical properties of the neutrosophic exponential model are described in detail. The estimation of a neutrosophic parameter by the method of maximum likelihood is discussed and illustrated with examples. The suggested neutrosophic exponential distribution (NED) model involves the interval time it takes for certain particular events to occur. Thus, the proposed model may be the most widely used statistical distribution for the reliability problems. For conceptual understanding, a wide range of applications of the NED in reliability engineering is given, which indicates the circumstances under which the distribution is suitable. Furthermore, a simulation study has been conducted to assess the performance of the estimated neutrosophic parameter. Simulated results show that imprecise data with a larger sample size efficiently estimate the unknown neutrosophic parameter. Finally, a complex dataset on remission periods of cancer patients has been analyzed to identify the importance of the proposed model for real-world case studies.
\end{abstract}

\section{Introduction}

One of the most common continuous distributions is the exponential distribution. The exponential model can be considered as a continuous description of the geometric distribution [1]. For reliability concerns, the exponential distribution is probably the most commonly used statistical distribution. The exponential distribution is especially appropriate and easier to implement in engineering to model the existence of nonwearing materials [2]. For specific measurement variables, such as interarrival time (IAT) and time to failure (TTF), an exponential model provides the best distributional postulate [3]. The IAT variable is derived from counting processes and represents the space (or time) between two adjacent events. For example, waiting lines, communication networks, and industrial processes with congested segments are popular applications [4]. The IAT is exactly exponential when the underlying counting process is
Poisson, so the two models are identical representations of the process. The TTF variable is used in component or system reliability analysis [5]. Because of its memoryless property, the exponential distribution is often used extensively in reliability theory. The exponential distribution models the TTF well when these components are subjected to the constant hazard of randomly occurring failurecausing loads [6]. The expectation of a constant rate is rarely met in real-world scenarios. Most engineering systems, such as electronic devices, are prone to wear, meaning their life history influences the failure hazard [7]. As a result, over the life cycle of a more general object, the hazard function is not constant [8]. However, the hazard function is constant for some types of systems in engineering applications [9]. This category includes the majority of electronic components. Most systems (particularly electronic ones) have a life cycle that follows the bathtub curve $[10,11]$. It is a good fit for modeling the steady hazard rate part of the bathtub curve in 
reliability studies. Exponential variables may also be employed to describe scenarios in which events happen with a fixed probability per unit distance, such as the length of a DNA strand between mutations or on a given lane, the gap between road kills, and so on $[12,13]$. The IAT, that is, the time between clients joining the system, are frequently modeled as an exponentially followed variable in queuing theory. In physics, the heights of the different molecules in a gas at a steady pressure and temperature in a uniformly gravitational environment adopt an estimated exponential distribution [14]. The exponential distribution is often applied in hydrology to look at extreme values of annual or monthly maximum river discharge volumes and total rainfall [15].

In this work, a new generalization of the exponential distribution is suggested in the hopes of expanding its applicability in the field of reliability modeling. This generalization is based on the notion of neutrosophy presented by Smarandache [16]. The analysis of false or true statements, but indeterminate, neutral, inconsistent, or something in between, is oriented by neutrosophy logic [17]. Every area has its neutrosophic component, namely, the indeterminacy part, on the mathematical side. Smarandache made the first effort to use the neutrosophic approach in statistics, precalculus, and calculus to cope with imprecision in study variables [18]. As a result, neutrosophic statistics have given rise to research topics that deal with the effect of indeterminacy in statistical modeling. Some recent literature has recently made the first step toward describing the neutrosophic principle of statistical modeling [19-21]. Neutrosophic measures probability and descriptive statistical are discussed in [22]. Neutrosophic decision-making applications in quality control seem to be very efficient [23]. Salama et al. first looked at the neutrosophic algebraic structures of probability distributions [24]. Nevertheless, works focusing on neutrosophic statistics have always relied on the applications side of the neutrosophic logic, and algebraic structures of probability distributions have rarely been addressed.

In this study, the notion of the NED has been described with the primary goal of incorporating vague information about the study variables. Vagueness in study parameters cannot be overlooked for practical analyses and should be integrated with the model being employed to describe a system. To the best of our knowledge, no published study has addressed the neutrosophic calculus of the exponential model. As a result, it is one of the factors that motivate us forward in our work.

The remainder of this study is outlined as follows. The NED and some of its useful functions are described in Section 2. Section 3 demonstrates some examples of the NED. The method estimation for the parameter of NED is provided in Section 4. The quantile function of NED is discussed in Section 5. A simulation study for demonstrating the performance of the neutrosophic estimator is carried out in Section 6. Implementation procedure of the NED for the real-world problem is given in Section 7. Finally, Section 8 concludes the research outcomes.

\section{Proposed Model and Some Useful Reliability Measures}

The neutrosophic random variable $Z$, which equals the distance between successive events in a Poisson process, follows the NED model with the following neutrosophic density function $\left(\mathrm{PDF}_{\mathrm{N}}\right)$ :

$$
\varphi_{N}(z)=\theta_{N} \exp \left(-z \theta_{N}\right) I_{(0, \infty)}(z), \quad z>0 .
$$

where $\theta_{N} \varepsilon\left[\theta_{L}, \theta_{U}\right]$ and $Z$ is a nonnegative variable usually denotes the working time, age, or life expressed in miles, hours, rounds, cycles, and so on, in reliability studies.

The region under this function curve, say $z_{1}$ to $z_{2}$ directly, represents the probability that an item randomly selected from the NED would fail during this period. Figure 1 shows the form of the distribution with the neutrosophic parameter $\theta_{N}=[0.5,1.5]$ if the data are believed to be NED.

Figure 1 shows the neutrosophic area because of the indeterminate value of the failure rate parameter $\theta_{N}$. Some other useful functions of the NED can be developed in the forms of the following theorems.

Theorem 1. The area under the curve characterized by the NED is one.

Proof. Let $\varphi: \mathrm{R} \longrightarrow \mathrm{P}(\mathrm{R})$ be a continuous neutrosophic exponential function concerning range such that

$$
\phi(z)=\left[\phi_{L}(z), \phi_{U}(z)\right], \quad 0<z<\infty,
$$

where $\varphi(z)$ is a sturdy curve, as represented by Figure 1 .

The neutrosophic area of the sturdy curve corresponds to

$$
\begin{aligned}
A_{N} & =\int_{0}^{\infty} \varphi_{N}(z) \mathrm{d} z \\
& =\int_{0}^{\infty}\left[\varphi_{L}(z), \varphi_{U}(z)\right] \mathrm{d} z \\
& =\left[\int_{0}^{\infty} \varphi_{L}(z) \mathrm{d} z, \int_{0}^{\infty} \varphi_{U}(z) \mathrm{d} z\right] \\
& =[a, b] .
\end{aligned}
$$

$a=\int_{0}^{\infty} \theta_{L} \exp \left(-z \theta_{L}\right) \mathrm{d} z=1$ and $b=\int_{0}^{\infty} \theta_{U} \exp \left(-z \theta_{U}\right) \mathrm{d} z=$ 1 , hence proved.

Theorem 2. The neutrosophic reliability function of the NED is $\left[\exp \left(-z \theta_{L}\right), \exp \left(-z \theta_{U}\right)\right]=\exp \left(-z \theta_{N}\right)$. 
Proof. The neutrosophic reliability function of the NED is determined by

$$
\begin{aligned}
\omega_{N}(z) & =P[Z>z] \\
& =1-P[Z<z] \\
& =1-\int_{0}^{z} \varphi_{N}(z) \mathrm{d} z \\
& =1-\int_{0}^{z}\left[\varphi_{L}(z), \varphi_{U}(z)\right] \mathrm{d} z \\
& =\left[1-\int_{0}^{z} \varphi_{L}(z) \mathrm{d} z, 1-\int_{0}^{z} \varphi_{U}(z) \mathrm{d} z\right] \\
& =[c, d],
\end{aligned}
$$

where $c=1-\int_{0}^{z} \phi_{L}(z) \mathrm{d} z=\exp \left(-z \theta_{L}\right)$ and $d=1-\int_{0}^{z} \phi_{U}$ $(z) \mathrm{d} z=\exp \left(-z \theta_{U}\right)$, hence proved.

The function $\omega_{N}(z)$ is most useful in reliability analysis, which establishes a connection between a unit's age and the likelihood of that unit surviving to that age while beginning the work at zero. The function $\omega_{N}(z)$ of the NED with neutrosophic parameter $\theta_{N}=[0.5,1.5]$ would be shaped as given in Figure 2. The failure neutrosophic rate function, conditional neutrosophic function, and $\mathrm{PDF}_{\mathrm{N}}$ all can be determined using this function.

Corollary 1. The hazard function $h_{N}(z)$ of the NED is $\theta_{N}$.

Proof. The ratio of $\varphi_{N}(z)$ to $\omega_{N}(z)$ results in the desired $h_{N}(z)$.

Corollary 2. The distribution function $\left(\Phi_{N}(z)\right)$ of the NED is $1-\exp \left(-z \theta_{N}\right)$.

Proof. The result of the distribution function is obtained by solving the following expression:

$$
\begin{aligned}
\Phi_{N}(z) & =\int_{0}^{z} \varphi_{N}(z) \mathrm{d} z \\
& =1-\exp \left(-z \theta_{N}\right) .
\end{aligned}
$$

Thus, in neutrosophy philosophy, the hazard rate for an object of a given age $(z)$ is referred to as an interval rate of death.

Theorem 3. The median of the NED is $\left[\left(\ln (2) / \theta_{U}\right),\left(\ln (2) / \theta_{L}\right)\right]$.

Proof. Neutrosophic median $\left(M_{N}\right)$ is the solution of the following expression:

$$
\begin{array}{r}
\int_{0}^{M_{N}} \Phi_{N}(z) \mathrm{d} z=\left[\frac{1}{2}, \frac{1}{2}\right], \\
{\left[\int_{0}^{M_{N}} \Phi_{L}(z) \mathrm{d} z, \int_{0}^{M_{N}} \Phi_{U}(z) d\right]=\left[\frac{1}{2}, \frac{1}{2}\right],}
\end{array}
$$

where $\Phi_{L}(z)=1-\exp \left(z \theta_{L}\right)$ and $\Phi_{U}(z)=1-\exp \left(z \theta_{U}\right)$.

Analytical simplification of (6) implies

$$
\begin{aligned}
& M_{N} \theta_{L}=\ln (2), \\
& M_{N} \theta_{U}=\ln (2),
\end{aligned}
$$

implying thereby

$$
M_{N}=\left[\frac{\ln (2)}{\theta_{U}}, \frac{\ln (2)}{\theta_{U}}\right]
$$

Corollary 3. First quantile $\left(Q_{I N}\right)$ and the third quantile $\left(Q_{3 N}\right)$ of the $N E D$ are $\left[\left(\ln (4 / 3) / \theta_{U}\right),\left(\ln (4 / 3) / \theta_{L}\right)\right]$ and $\left[\left(\ln (4) / \theta_{U}\right),\left(\ln (4) / \theta_{L}\right)\right]$, respectively.

Proof. $\mathrm{Q}_{\mathrm{IN}}$ and $\mathrm{Q}_{3 \mathrm{~N}}$ by definition are corresponded to solutions such that

$$
\begin{aligned}
& \int_{0}^{Q_{I N}} \Phi_{N}(z) \mathrm{d} z=\left[\frac{1}{4}, \frac{1}{4}\right], \\
& \int_{0}^{Q_{3 N}} \Phi_{N}(z) \mathrm{d} z=\left[\frac{3}{4}, \frac{3}{4}\right] .
\end{aligned}
$$

Therefore, following Theorem 3, we can write

$$
\begin{aligned}
Q_{I N} & =\left[\frac{\ln (4)}{\theta_{U}}, \frac{\ln (4)}{\theta_{L}}\right], \\
Q_{3 N} & =\left[\frac{\ln (4 / 3)}{\theta_{U}}, \frac{\ln (4 / 3)}{\theta_{L}}\right] .
\end{aligned}
$$

Theorem 4. The neutrosophic average time to failure of the $N E D$ is $1 / \theta_{N}$.

Proof. The neutrosophic average time to failure is determined as

$$
\begin{aligned}
\operatorname{MTT}_{N} & =\int_{0}^{\infty} \omega_{N}(z) \mathrm{d} z \\
& \left.=\int_{0}^{\infty}\left[\omega_{U}(z), \omega_{L}(z)\right] \mathrm{d} z \text { (see Figure } 2\right) \\
& =\int_{0}^{\infty} \exp \left(-z \theta_{U}\right) \mathrm{d} z, t \int_{0}^{\infty} \exp \left(-z \theta_{L}\right) \mathrm{d} z \\
& =\left[\frac{1}{\theta_{U}}, \frac{1}{\theta_{L}}\right] \\
& =\frac{1}{\theta_{N}} .
\end{aligned}
$$

Theorem 5. The variance of the NED is $1 / \theta_{N}^{2}$.

Proof. By definition, variance is

$$
\sigma_{N}^{2}(Z)=E\left(Z^{2}\right)-\left(\mathrm{MTT}_{N}\right)^{2}
$$

where $\sigma_{N}^{2}(Z)$ stands for neutrosophic variance.

Now, 


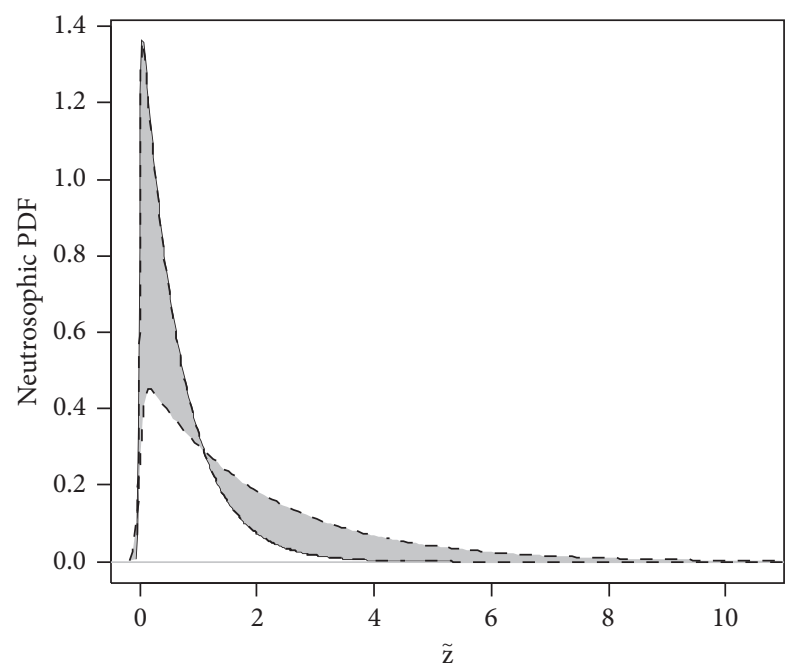

Figure 1: The sturdy curve of exponential distribution.

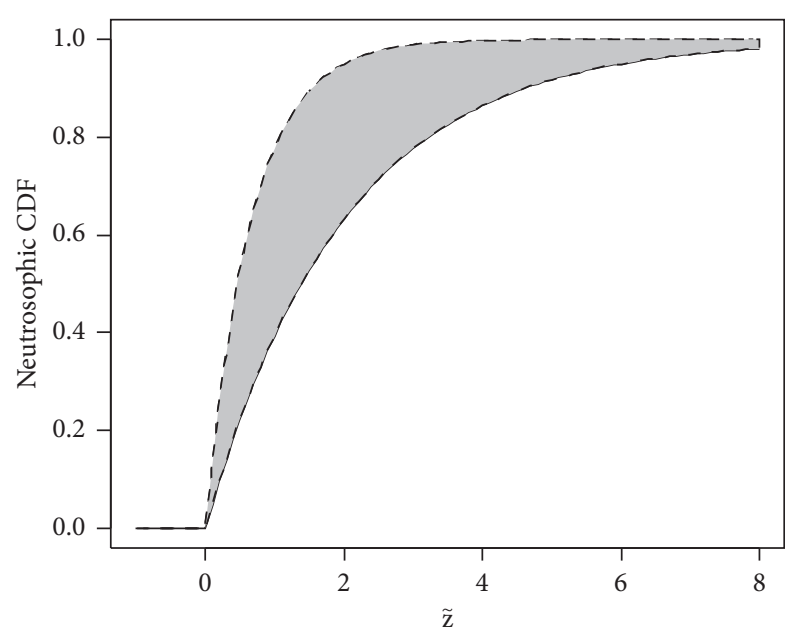

Figure 2: The function $\omega_{N}(z)$ for the NED.

$$
E\left(Z^{2}\right)=\int_{0}^{\infty} z^{2} \varphi_{N}(z) \mathrm{d} z
$$

Since $\varphi_{N}(z)=-\omega_{N}(z)$, we have the following:

$$
\begin{aligned}
E\left(Z^{2}\right) & =\frac{2}{\theta_{N}} \int_{0}^{\infty} \omega_{N}(z) \mathrm{d} z \\
& =\frac{2}{\theta_{N}} \int_{0}^{\infty}\left[\omega_{U}(z), \omega_{L}(z)\right] \mathrm{d} z \\
& =\frac{2}{\theta_{N}}\left[\int_{0}^{\infty} \exp \left(-z \theta_{U}\right) \mathrm{d} z, \int_{0}^{\infty} \exp \left(-z \theta_{L}\right) \mathrm{d} z\right] \\
& =\frac{2}{\theta_{N}}\left[\frac{1}{\theta_{U}}, \frac{1}{\theta_{L}}\right] \\
& =\left[\frac{2}{\theta_{U}^{2}}, \frac{2}{\theta_{L}^{2}}\right] .
\end{aligned}
$$

Thus, (12) yields

$$
\sigma_{N}^{2}(Z)=\left[\frac{2}{\theta_{U}^{2}}, \frac{2}{\theta_{L}^{2}}\right]-\left(\left[\frac{1}{\theta_{U}}, \frac{1}{\theta_{L}}\right]\right)^{2}
$$

and simplifying (15) provides

$$
\sigma_{N}^{2}(Z)=\left[\frac{1}{\theta_{U}^{2}}, \frac{1}{\theta_{L}^{2}}\right]
$$

Likewise, the other properties of the NED can be established in a neutrosophic environment. Some applications of the proposed model are presented to understand the initial concepts derived for the NED.

\section{Illustrative Examples}

In this section, the notion of the NED has been described with a series of examples in the area of reliability studies.

Example 1. The lifetime of a certain mechanical component follows the NED with the indeterminate failure rate $\theta_{N}=$ $\left[5.26 \times 10^{-5}, 8.70 \times 10^{-5}\right]$ per hour. In continuous operation, find the probability that the component survives for three months. Also, find the meantime to failure of this component.

Solution 1. From (1), the neutrosophic reliability function of the NED is

$$
\omega_{N}(z)=\exp \left(-z \theta_{N}\right)
$$

and the probability that the component survives for three months ( $z=2190$ hours)is given by

$\omega_{N}(z)=\exp \left(-2190\left[5.26 \times 10^{-5}, 8.70 \times 10^{-5}\right]\right)=[0.8265,0.8911]$.

Hence, the chance of surviving the component without failure for a period of three months is [0.8265, 0.8911].

Now, from (11), 


$$
\operatorname{MTT}_{N}=\left[\frac{1}{\theta_{U}}, \frac{1}{\theta_{L}}\right]
$$

Thus,

$$
\begin{aligned}
& =\left[\frac{1}{8.70 \times 10^{-5}}, \frac{1}{5.26 \times 10^{-5}}\right] \\
& =[11494.25,19011.41] \text { hours } \approx[15.7,26.0] \text { months. }
\end{aligned}
$$

Example 2. Failure mechanism of the alternators used in automobiles follows the NED for an average lifespan of $[8,12]$ years. Mr. Adnan buys a six-year-old car with a functioning alternator to keep it for eight years. Determine the probability of the alternator failing during his possession.

Solution 2. Let $Z$ denote the neutrosophic random variable that follows NED.

Given that $\operatorname{MTT}_{N}=\left[\left(1 / \theta_{U}\right),\left(1 / \theta_{L}\right)\right]=[8,12]$ years, this implies $\left[\theta_{L}, \theta_{U}\right]=[0.083,0.125]$.

Now, the required probability is

$$
\begin{aligned}
P[Z<8] & =\Phi_{N}(8)(\text { corollary } 2) \\
& =[0.079,0.117] .
\end{aligned}
$$

Thus, the chance that the alternator fails during his ownership is approximated by $[8,12] \%$.

\section{Parameter Estimation}

The method for estimating the parameter of the NED, namely, neutrosophic maximum likelihood estimation (NML), has been introduced. Let $n$ sample $\left\{X_{i}, i=1,2, \ldots, n\right\}$ values be taken from the NED. The question is, which value of the neutrosophic parameter should be used for the observed sample? This value can be determined by the likelihood function of the neutrosophic model. As neutrosophy exists in the parameter of the NED, therefore, NML function of the NED is given by

$$
\varpi_{N}\left(z, \theta_{N}\right)=n \log \theta_{N}-\theta_{N} \sum{ }_{i}^{n} z_{i},
$$

and the NML estimates, namely, $\widehat{\theta}_{L}$ and $\widehat{\theta}_{U}$, can be obtained by solving the following expression:

$$
\frac{\delta \oplus_{N}\left(z, \theta_{N}\right)}{\delta \theta_{N}}
$$

Using the neutrosophic calculus [18], (23) yielded

$$
=\left[\frac{\delta \varpi_{L}\left(z, \theta_{L}\right)}{\delta \theta_{U}}, \frac{\delta \varpi_{U}\left(z, \theta_{U}\right)}{\delta \theta_{L}}\right],
$$

where $\varpi_{L}\left(z, \theta_{L}\right)=n \log \theta_{L}-\theta_{L} \sum_{i}^{n} z_{i}$ and $\varpi_{N}\left(z, \theta_{U}\right)=$ $n \log \theta_{U}-\theta_{U} \sum_{i}^{n} z_{i}$.

Simplification of (24) provides

$$
\frac{\delta \oplus_{N}\left(z, \theta_{N}\right)}{\delta \theta_{N}}=\left[\frac{n}{\theta_{L}}-\sum_{i}^{n} z_{i}, \frac{n}{\theta_{U}}-\sum_{i}^{n} z_{i}\right] .
$$

Setting (25) equating to $[0,0]$ provides

$$
\begin{aligned}
& \widehat{\theta}_{L}=\frac{n}{\sum_{i}^{n} z_{i}}, \\
& \widehat{\theta}_{U}=\frac{n}{\sum_{i}^{n} z_{i}} .
\end{aligned}
$$

Thus, $\widehat{\theta}_{N}=\left[\widehat{\theta}_{L}, \widehat{\theta}_{U}\right]=\left(n / \sum_{i}^{n} z_{i}\right)$, which is a single crisp value and coincides with the classical MLE.

However, if imprecision in the observed data $(\widetilde{z})$ is considered; then NML of the neutrosophic parameter would be modified as

$$
\widehat{\theta}_{N}=\left[\widehat{\theta}_{L}, \widehat{\theta}_{U}\right]=\left[\frac{n}{A}, \frac{n}{B}\right]
$$

where

$$
\begin{aligned}
& A=\min _{\tilde{z}}=\text { sum of the minmum values of the neutrosophic dataset, } \\
& B=\max _{\widetilde{z}}=\text { sum of the maximum values of the neutrosophic dataset. }
\end{aligned}
$$

An intuitive explanation of the NML estimation could be described with the following example.

Example 3. Assume that the life (in years) of the Philips brand refrigerator compressor is best described by the NED with unknown parameter. Five compressors have been independently tested, and their lifetimes are determined to be $4,3.5,3.9,4.2,4.5$, and 4.7 years, respectively. What is the NML estimate of $\theta_{N}$ ?

Solution 3. The joint density is the product of the individual densities since compressor lifetimes are independently tested. Using (22), the log of the NML function is therefore given by

$$
\varpi_{N}\left(z, \theta_{N}\right)=5 \log \theta_{N}-24.8 \theta_{N} .
$$

Finally, simplifying (29) leads to the NML estimate of $\theta_{N}$ as

$$
\widehat{\theta}_{N}=\frac{6}{24.8}=0.24 \text { year. }
$$

Example 4. Now, we assume that the life (in years) of the Philips brand refrigerator compressor is best described by the NED with an unknown constant failure rate; that is, $\theta_{L}=\theta_{U}=\theta$. Five compressors have been independently tested, and their lifetimes are determined to be 4, 3.5, [3.9, $4.1], 4.2,[4.3,4.6]$, and 4.7 years, respectively. What is the NML estimate of $\theta_{N}$ ? 
Solution 4. Here, the indeterminacies are recorded in the lifetime values of the third and fifth components; that is, values in interval implies exactly values of these components are not precisely recorded.

Now, considering the neutrosophy in observed data, the lives of all these components are given by

$$
\sum_{i}^{n} z_{i}=[24.6,25.1] \text { years. }
$$

Using (26), the NML estimate of $\theta_{N}$ is given by

$$
\widehat{\theta}_{N}=[0.23,0.24] \text { year. }
$$

\section{Model Validation}

The neutrosophic quantile function $\left(\mathrm{QF}_{N}\right)$ of NED can be found by solving the following expression for $z$ :

$$
Z_{j}=\Phi^{-1}\left(\xi_{j}\right)
$$

In the case of NED,

$$
Z_{j}=-\frac{1}{\theta_{N}} \ln \left(1-\xi_{j}\right), \quad j=1,2, \ldots,
$$

where $\xi_{j}$ follows a uniform distribution with parameters 0 and 1 ; that is, $\xi_{j} \sim U[0,1]$.

Statistically speaking, the $\mathrm{QF}_{N}$ are used to establish quantile analogs of standard moment-based descriptive measures and expand those measures. This function can be used to produce random data that describes the density given in (1). Analytical results of mean, variance, and all other properties can be validated using the Carlo simulation.

In R software, the NED can be easily simulated to view the validity of theory-based derived results. Set $\theta_{N}=[2,4]$ in the NED and 10000 samples are randomly generated from the $U[0,1]$. Using (22), 10000 pseudo neutrosophic random samples are generated from the NED. These simulated data utilized to validate the analytical properties as discussed in Section 2 are investigated. The exact results for the mean, variance, and quantiles of the NED alongside the simulated values are given in Table 1.

Table 1 shows that the descriptive measures employing simulation are very close to the exact quantities, suggesting that the simulation produces results identical to the expected results described in Section 2. As a result, we can deduce that the estimates of other parameters computed with the simulated data would inevitably equal the true parameters.

\section{Simulation Study}

In this section, the performance of the NML estimator has been assessed in terms of the neutrosophic average biased $\left(\mathrm{AB}_{N}\right)$ and neutrosophic root mean square error $\left(\mathrm{RMS}_{N}\right)$ as defined as follows [25]:
TABLE 1: Comparisons of simulated versus analytical results.

\begin{tabular}{lcc}
\hline Neutrosophic parameters & Exact results & Simulated results \\
\hline Mean & {$[0.250,0.500]$} & {$[0.249,0.499]$} \\
Variance & {$[0.063,0.250]$} & {$[0.062,0.246]$} \\
$Q_{I N}$ & {$[0.072,0.144]$} & {$[0.071,0.144]$} \\
$Q_{2 N}$ & {$[0.173,0.346]$} & {$[0.174,0.349]$} \\
$Q_{3 N}$ & {$[0.346,0.693]$} & {$[0.344,0.695]$} \\
\hline
\end{tabular}

$$
\begin{aligned}
\mathrm{AB}_{N} & =\frac{\sum_{j=1}^{N}\left(\hat{\theta}_{N j}-\theta_{N}\right)}{N}, \\
\mathrm{RMSE}_{N} & =\sqrt{\frac{\sum_{j=1}^{N}\left(\hat{\theta}_{N j}-\theta_{N}\right)^{2}}{N}} .
\end{aligned}
$$

A Monte Carlo simulation is run in $\mathrm{R}$ software with various sample sizes and fixed value of the neutrosophic parameter $\theta_{N}=[2,4]$. An imprecise dataset is generated using the NED with $\theta_{N}=[2,4]$, and simulation analysis is replicated for a total of $N=10000$ times with sample sizes of $n=10,20,30$, and 50, respectively. The performance measures of the NML estimator are then computed and given in Table 2.

It can be seen from the results, as the sample size $n$ goes up, the $A B_{N}$ and $\mathrm{RMSE}_{\mathrm{N}}$, that is, biases, decrease. Thus, the study concludes that the NML estimator provides reliable estimation with a larger sample size.

\section{Real-World Application}

In this section, a practical application using a real-world dataset has been used in order to assess the interest in the NED model. The data under consideration includes a set of remission periods in months from 128 cancer patients. The remission periods are based on a subset of data reported by Lee and Wang [26] from bladder cancer research and are considered here solely for descriptive purposes. According to the findings of the goodness of fit test rooted on asymptotic likelihood function, the exponential distribution is one of the plausible models for the remission times. The appropriateness of the exponential model can be seen in Figure 3.

Figure 3 provides a quick graphical summary of the exponential model on the remission time dataset. In Figure 3, the PP-plot and CDF-plot demonstrate that the exponential model well fits remission time data reported for cancer patients. Initially, data are the crisp measurements; however, for the sake of illustration, we treat them as indeterminate sample values for certain cancer patients, as indicated in Table 3.

Table 3 shows that remission times for certain cancer patients, such as [7.26, 8.2], [12, 14.77], [15, 17.2], [5.3, 7.1], $[75.02,81]$, and $[1.5,3.2]$, are not precisely reported but are provided in intervals. These ambiguities or uncertainties in the sample render the existing exponential model inapplicable. On the contrary, the suggested NED may effectively be used to investigate the properties of this neutrosophic dataset. The descriptive statistics of the remission time data using the NED model are given in Table 4. 
TABLE 2: Performance of NML estimate of the NED for simulated neutrosophic data.

\begin{tabular}{lrr}
\hline Sample size & $\mathrm{AB}_{N}$ & \multicolumn{1}{c}{$\mathrm{RMSE}_{N}$} \\
\hline 10 & {$[0.222,0.443]$} & {$[0.822,1.645]$} \\
20 & {$[0.104,0.207]$} & {$[0.506,1.011]$} \\
30 & {$[0.066,0.133]$} & {$[0.394,0.788]$} \\
50 & {$[0.039,0.078]$} & {$[0.295,0.590]$} \\
\hline
\end{tabular}

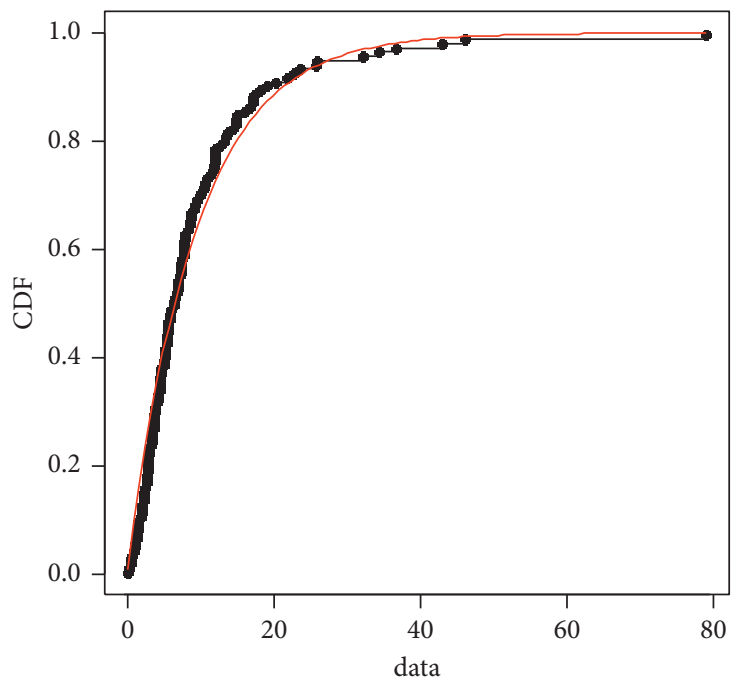

(a)

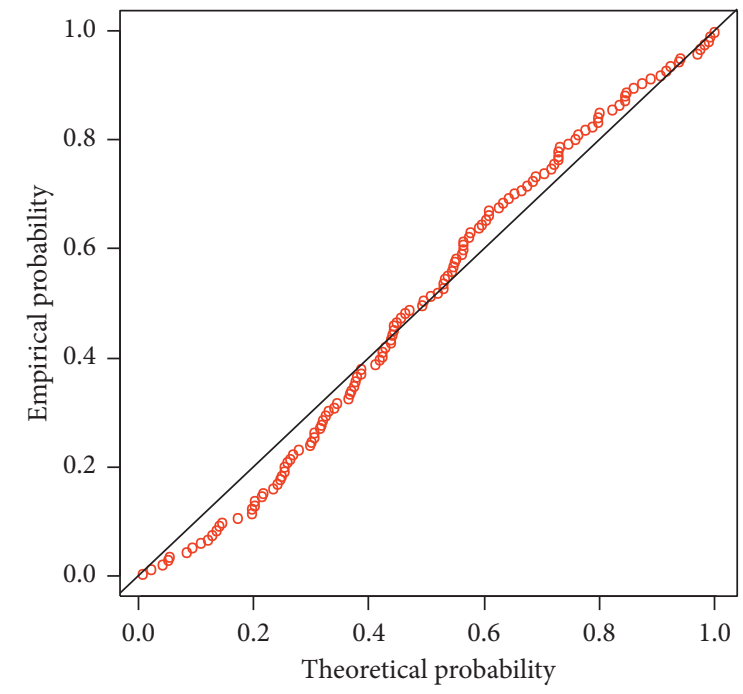

(b)

FIGURE 3: Graphical overview of the remission time dataset: (a) CDF-plot and (b) PP-plot.

TABLE 3: Remission periods of 128 cancer patients.

\begin{tabular}{lcccccccccccc}
\hline \multicolumn{10}{c}{ Remission times } \\
\hline 0.08 & 2.09 & 3.48 & 4.87 & 6.94 & 8.66 & 13.11 & 23.63 & 0.2 & 2.23 & 3.52 & 4.98 & 6.97 \\
9.02 & 13.29 & 0.4 & 2.26 & 3.57 & 5.06 & 7.09 & 9.22 & 13.8 & 25.74 & 0.5 & 2.46 & 3.64 \\
5.09 & {$[\mathbf{7 . 2 6 , 8 . 2 ]}$} & 9.47 & 14.24 & 25.82 & 0.51 & 2.54 & 3.7 & 5.17 & 7.28 & 9.74 & 14.76 & {$[5.3,7.1]$} \\
0.81 & 2.62 & 3.82 & 5.32 & 7.32 & 10.06 & {$[\mathbf{1 2}, \mathbf{1 4 . 7 7}]$} & 32.15 & 2.64 & 3.88 & 5.32 & 7.39 & 10.34 \\
14.83 & 34.26 & 0.9 & 2.69 & 4.18 & 5.34 & 7.59 & 10.66 & 15.96 & 36.66 & 1.05 & 2.69 & 4.23 \\
5.41 & 7.62 & 10.75 & 16.62 & 43.01 & 1.19 & 2.75 & 4.26 & 5.41 & 7.63 & {$[\mathbf{1 5}, \mathbf{1 7 . 2}]$} & 46.12 & 1.26 \\
2.83 & 4.33 & 5.49 & 7.66 & 11.25 & 17.14 & {$[\mathbf{7 5 . 0 2 , 8 1}]$} & 1.35 & 2.87 & 5.62 & 7.87 & 11.64 & 17.36 \\
1.4 & 3.02 & 4.34 & 5.71 & 7.93 & 11.79 & 18.1 & 1.46 & 4.4 & 5.85 & 8.26 & 11.98 & 19.13 \\
1.76 & 3.25 & 4.5 & 6.25 & 8.37 & 12.02 & {$[\mathbf{1 . 5 , 3 . 2}]$} & 3.31 & 4.51 & 6.54 & {$[\mathbf{7 . 5}, \mathbf{8 . 2}]$} & 12.03 \\
20.28 & 2.02 & 3.36 & 6.76 & 12.07 & 21.73 & 2.07 & 3.36 & 6.93 & 8.65 & 12.63 & 22.69 \\
\hline
\end{tabular}

Bold values mean imprecise/indeterminate values.

TABle 4: Neutrosophic statistics of remission times data by using the proposed model.

\begin{tabular}{lc}
\hline Descriptive measures & \\
\hline Mean & {$[9.18,9.24]$} \\
Variance & {$[84.29,85.39]$} \\
Median & {$[6.40,6.36]$} \\
First quantile & {$[2.64,2.71]$} \\
Third quantile & {$[12.12,12.71]$} \\
\hline
\end{tabular}

The results in Table 4 demonstrate that the critical numerical statistics of the remission times data are given in ranges due to certain uncertainties in the observed sample. Consequently, the suggested model may be used to analyze data that is expected to follow the NED.

\section{Conclusions}

A new representation of the exponential statistical model referred to as the NED has been proposed in this work. Various structural properties of the proposed model under the neutrosophic environment are extensively discussed. The analytical expressions for neutrosophic mean, neutrosophic variance, neutrosophic median, and other related quantities are derived. The NED best describes failure patterns of many in-service components. The estimation method considering vagueness in the observed data has been established and explained with examples. The analytical results of the proposed NED are validated using the notion of the neutrosophic quantile function. A simulation study has been conducted to validate the performance of the estimated 
neutrosophic parameter. The simulated findings show that indeterminate sample data with a large size efficiently estimate the unknown parameter. Some applicability examples of the NED mainly for the processing indeterminacies in lifetime data are considered.

8.1. Further Works. We believe that the neutrosophic generalization of the classical model may broaden the scope of NED in survival and reliability studies.

\section{Data Availability}

All the data used for analysis and supporting results are given within the article.

\section{Conflicts of Interest}

The authors declare that they have no conflicts of interest.

\section{References}

[1] R. D. Gupta and D. Kundu, "Generalized exponential distribution: different method of estimations," Journal of Statistical Computation and Simulation, vol. 69, pp. 315-337, 2007.

[2] P. Celis, R. de la Cruz, C. Fuentes, and H. W. Gómez, "Survival and reliability analysis with an epsilon-positive family of distributions with applications," Symmetry, vol. 13, no. 5, p. 908, 2021.

[3] K. Das, "A comparative study of exponential distribution vs Weibull distribution in machine reliability analysis in a CMS design," Computers \& Industrial Engineering, vol. 54, no. 1, pp. 12-33, 2008.

[4] S. Nadarajah and F. Haghighi, "An extension of the exponential distribution," Statistics, vol. 45, no. 6, pp. 543-558, 2011.

[5] R. D. Gupta and D. Kundu, "Generalized exponential distribution: existing results and some recent developments," Journal of Statistical Planning and Inference, vol. 137, no. 11, pp. 3537-3547, 2007.

[6] Y. M. Low, "A new distribution for fitting four moments and its applications to reliability analysis," Structural Safety, vol. 42, pp. 12-25, 2013.

[7] N. Gorjian, L. Ma, M. Mittinty, P. Yarlagadda, and Y. Sun, "A review on degradation models in reliability analysis," in Engineering Asset Lifecycle Management, Proceedings of the 4th World Congress on Engineering Asset Management, pp. 369-384, Springer, London, UK, 2009.

[8] R. A. Chechile, "Mathematical tools for hazard function analysis," Journal of Mathematical Psychology, vol. 47, no. 5-6, pp. 478-494, 2003.

[9] M. S. Goodman, Y. Li, and R. C. Tiwari, "Detecting multiple change points in piecewise constant hazard functions," Journal of Applied Statistics, vol. 38, no. 11, pp. 2523-2532, 2011.

[10] G. Klutke, P. C. Kiessler, and M. A. Wortman, “A critical look at the bathtub curve," IEEE Transactions on Reliability, vol. 52, no. 1, pp. 125-129, 2003.

[11] W. J. Roesch, "Using a new bathtub curve to correlate quality and reliability," Microelectronics Reliability, vol. 52, no. 12, pp. 2864-2869, 2012.
[12] T. Hels and E. Buchwald, "The effect of road kills on amphibian populations," Biological Conservation, vol. 99, no. 3, pp. 331-340, 2001.

[13] M. E. Allentoft, M. Collins, D. Harker et al., "The half-life of DNA in bone: measuring decay kinetics in 158 dated fossils," Proceedings of the Royal Society B: Biological Sciences, vol. 279, no. 1748, pp. 4724-4733, 2012.

[14] W. Xie, Q. Xie, H. Zhou, and Z. Ren, "An exponential distribution scheme for the two-way coupling in transported PDF method for dilute spray combustion," Combustion Theory and Modelling, vol. 24, no. 1, pp. 105-128, 2020.

[15] I. E. Okorie, A. C. Akpanta, J. Ohakwe, and D. C. Chikezie, "The Extended Erlang-truncated exponential distribution: properties and application to rainfall data," Heliyon, vol. 3, p. e00296, Article ID e00296, 2017.

[16] F. Smarandache, Unifying A Field in Logics, Neutrosophy: Neutrosophic Probability; Set and Logic, American Research Press, Rehoboth, NM, USA, 1999.

[17] Z. Khan, M. Gulistan, N. Kausar, and C. Park, "Neutrosophic Rayleigh model with some basic characteristics and engineering applications," IEEE Access, vol. 9, pp. 71277-71283, 2021.

[18] F. Smarandache and H. E. Khalid, Neutrosophic Precalculus and Neutrosophic Calculus, PONS, Stuttgart, Germany, 2nd edi edition, 2018.

[19] Z. Khan, M. Gulistan, W. Chammam, S. Kadry, and Y. Nam, "A new dispersion control chart for handling the neutrosophic data," IEEE Access, vol. 8, pp. 96006-96015, 2020.

[20] Z. Khan, M. Gulistan, R. Hashim, N. Yaqoob, and W. Chammam, "Design of S-control chart for neutrosophic data: an application to manufacturing industry," Journal of Intelligent and Fuzzy Systems, vol. 38, no. 4, pp. 4743-4751, 2020.

[21] M. Aslam, N. Khan, and M. Khan, "Monitoring the variability in the process using neutrosophic statistical interval method," Symmetry, vol. 10, no. 11, p. 562, 2018.

[22] F. A Smarandache, Unifying Field in Logics: Neutrosophic Logic. Neutrosophy, Neutrosophic Set, Neutrosophic Probability and Statistics, InfoLearnQuest, Ann Arbor, MI, USA, 6th edition, 2007.

[23] M. Aslam, "A new sampling plan using neutrosophic process loss consideration," Symmetry, vol. 10, no. 5, p. 132, 2018.

[24] I. M. Hanafy, A. A. Salama, and K. Mahfouz, "Correlation of neutrosophic data," International Journal of Engineering Science, vol. 1, pp. 39-43, 2012.

[25] S. Wang and W. Gui, "Corrected maximum likelihood estimations of the lognormal distribution parameters," Symmetry, vol. 12, no. 6, p. 968, 2020.

[26] E. T. Lee and J. Wang, Statistical Methods for Survival Data Analysis, Vol. 476, John Wiley \& Sons, Hoboken, NJ, USA, 2003. 